Zeitschriftenartikel:

Begutachtet

Redaktion und Begutachtung:

Nele Heise (iD)

Digital Media \& Communication

Researcher Hamburg

Nils Zurawski (DD

Universität Hamburg

Erhalten: 01. Dezember 2019

Akzeptiert: 29. September 2020

Publiziert: 15. Dezember 2020

Lizenz:

(c) Rebeccca Moltmann

Dieses Werk steht unter einer Lizenz

Creative-Commons-Namensnennung 4.0

(CC-BY 4.0) International

(C) $($ i)

Datenverfügbarkeit:

Alle relevanten Daten befinden sich innerhalb der Veröffentlichung.

Interessenskonfliktstatement:

Die Autor:innen erklären, dass ihre Forschung ohne kommerzielle oder finanzielle Beziehungen durchgeführt wurde, die als potentielle

Interessenskonflikte ausgelegt werden können.

Empfohlene Zitierung:

Moltmann, R. (2020). Vom "Verfertigen der Gedanken": Das Potential von Podcasts für die geisteswissenschaftliche Wissenschaftskommunikation. kommunikation@gesellschaft, 21(2). https://doi.org/10.15460/kommges. 2020.21.2.624

\section{Vom "Verfertigen der Gedanken"}

\section{Das Potential von Podcasts für die geisteswissenschaftliche Wissen- schaftskommunikation}

\author{
Rebecca Moltmann ${ }^{\mathrm{a} *}$ (D) \\ a Universität Bielefeld \\ * Korrespondenz: rebecca.moltmann@uni-bielefeld.de
}

\begin{abstract}
Für die Vermittlung geisteswissenschaftlicher Forschungsinhalte und -praktiken scheinen auf den ersten Blick andere Formate nötig zu sein als etwa für die Naturwissenschaften, da im Zentrum der Geisteswissenschaften vor allem das wenig visualisierbare Reflexionswissen steht. Ausgehend von dieser Prämisse wird die These entfaltet, dass Podcasts und der auditiven Wissensvermittlung besonderes Potential für geisteswissenschaftliche Wissenschaftskommunikation zukommt. Der Fokus liegt dabei auf der Literaturwissenschaft, da sie zum einen häufig als besonders abstraktes und wenig greifbares Fach angesehen wird. Zum anderen hat sich in der Forschung der letzten Jahre eine stärkere praxeologische Perspektive auf die Literaturwissenschaft als Disziplin entwickelt, die hilfreiche Ansätze für den hier verfolgten Argumentationszusammenhang bietet. Im Anschluss an die exemplarische Analyse zweier Podcastfolgen mit literaturwissenschaftlicher Perspektive, werden Wissenstransfer und die Partizipation an Wissen als zentrale Aspekte des Potentials von Podcasts für die geisteswissenschaftliche Wissenschaftskommunikation herausgestellt. Mit Blick auf die Wissenschaftsforschung lassen sich in diesem Zusammenhang erste tentative Ansätze für Podcasts als ,Tool' der Wissenschaftsforschung aufzeigen.
\end{abstract}

Schlagworte: Wissenschaftspodcasts, Wissenschaftskommunikation, Geisteswissenschaften, Literaturwissenschaft, Wissenschaftsforschung, Praktiken 


\section{Wissen ,zeigen' in vermeintlich zwei ,Kulturen': Sind Geisteswissenschaften schwieriger zu vermitteln als Natur- und Technikwissenschaften?}

Im vorliegenden Beitrag stehen Podcasts als vielversprechendes Format für die geisteswissenschaftliche Wissenschaftskommunikation im Zentrum. Damit wird keinesfalls das fachübergreifende Potential von Podcasts in Frage gestellt. Ausgangspunkt ist vielmehr die Beobachtung, dass geisteswissenschaftliche Themen und insbesondere Forschungspraktiken weniger sichtbar zu sein scheinen, wenn es um die allgemeine gesellschaftliche Vorstellung von ,Wissenschaft' geht. Unter Geisteswissenschaften werden hier, der Systematik Schäfers (Schäfer, 2018) folgend ${ }^{1}, 1$. Geschichte und Archäologie, 2. Sprach- und Literaturwissenschaften, 3. Philosophie, Ethik- und Religionswissenschaft, 4. Kunstwissenschaften und 5. Rechtswissenschaften gefasst.

Diese geringe Sichtbarkeit lässt sich schon anhand einer schlichten GoogleBildersuche mit dem Stichwort „Wissenschaftler:innen“ feststellen. Wenig überraschend erscheinen unzählige Bilder von Menschen in weißen Kitteln, in Laboren, mit Reagenzgläsern, Schutzbrillen oder Mikroskopen. Ähnlich steht es um wissenschaftliche Themen in massenmedialen Darstellungen: In Romanen, Filmen oder Serien spielt Wissenschaft durchaus häufig eine prominente Rolle. Wir lesen aber tendenziell von Rechtsmediziner:innen, sehen auch hier Forscher:innen im Umgang mit komplexen technischen Apparaten in ihren Laboren zu oder werden während Dungeons \& Dragons-Rollenspielen en passant über physikalische Phänomene aufgeklärt. Produktionen mit Philolog:innen oder Kunsthistoriker:innen in der Hauptrolle sind deutlich weniger breit vertreten. ${ }^{2}$

Dies lässt sich etwa mit der ,Fiction Database' des von der VW-Stiftung geförderten Forschungsprojekts „Fiction meets Science“ illustrieren (Projektleitung: Universität Bremen) ${ }^{3}$, in dessen Rahmen Wissenschaft und ihre fiktionale Bearbeitung im Zusammenspiel analysiert werden. In der besagten Datenbank befinden sich aktuell (Stand: 28. August 2020) 390 Titel, die nach verschiedenen Kategorien gefiltert werden können - dazu gehört die der „scientific discipline". Von den angegebenen 25 Auswahlfächern ist eines geisteswissenschaftlich, zwei weitere lassen sich immerhin in Teilen den Geisteswissenschaften zuordnen: „archaeology”, „natural history “ und „anthropology". Hier finden sich insgesamt 19 thematisch passende Treffer - von 390. Die Gründe für dieses Verhältnis können natürlich unterschiedlichster Natur sein und etwa in der Beschaffenheit der verschiedenen Teilprojekte oder dem Fokus der Studien liegen. Dennoch kann das Projekt als exemplarisch für die randständige mediale Repräsentation geisteswissenschaftlicher Themen und

\footnotetext{
1 Schäfer wiederum bezieht sich auf die Einordnung der Organisation for Economic Co-operation and Development (OECD) von 2007, ergänzt sie aber um die Rechtswissenschaften, die nach OECD den Sozialwissenschaften zufallen.

2 Vgl. hierzu etwa Milde (2009) und Hanel (1994), die feststellen, dass es in „Wissenschaftsfilmen [traditionell] um Themen aus Naturwissenschaft, Technik und Medizin“ gehe (Milde, 2009, S. 48).

3 https://www.fictionmeetsscience.org (Zugriff am 03.09.2020).
} 
Forschungspraktiken gelten (vgl. dazu auch Milde, 2009). ${ }^{4}$

Am Titel „Fiction meets Science“ lässt sich außerdem eine sprachliche Besonderheit zeigen, die die Grundlage eines ersten Interpretationsversuchs darstellt: Mit dem englischen science sind begriffsgeschichtlich betrachtet nur die Naturwissenschaften gemeint. Die humanities lassen sich nicht unter science subsumieren und führen diese auch nicht in einer Zusammensetzung mit, wie etwa die social sciences oder das deutsche ,Geisteswissenschaften' - im 19. Jahrhundert geprägt von Wilhelm Dilthey (1883). ${ }^{5}$ Hamann (2014) bezeichnet den „dezidiert akademische[n] Charakter der Geisteswissenschaften“ gar als „Spezifikum des deutschen Sprach- und Kulturraums“ (Hamann, 2014, S. 14).

Neben der wissenschaftshistorischen Entwicklung der Disziplinen hat nicht zuletzt die Rede von den ,zwei Kulturen', die auf Charles Percy Snow zurückgeht, die teilweise ,anti-podisch' anmutende Konstellation und das Verständnis der verschiedenen Wissenschaftsdisziplinen lange Zeit geprägt und konsolidiert.

Snow (1988) beschreibt etwa die Geisteswissenschaftler:innen als in die Vergangenheit blickend und tendenziell pessimistisch, die Naturwissenschaftler:innen als optimistischer und an der Zukunft und gesellschaftlichen Realität ausgerichtet. Die Abgrenzung von Geistes- und Naturwissenschaften (für die schon bei Snow am Rande angedeuteten, aber nicht in seine Abhandlung aufgenommenen Sozialwissenschaften als mögliche, dritte Kultur' gilt dies ebenso) ist längst nicht mehr zeitgemäß, nicht zuletzt aufgrund der Unterschiede, die es bereits zwischen vermeintlich benachbarten Disziplinen wie etwa Literaturwissenschaft und Linguistik geben kann - oder eben Gemeinsamkeiten in der Forschungspraxis weiter voneinander entfernter Fächer. „Was heute Fachkulturen ausmacht, ist also weniger ein angestammter Fachkanon als das lebendige, alltägliche fachliche Tun als doing discipline“, folgern Kaufmann, Satilmis \& Mieg (2019). Die dennoch bis heute auffällige Dominanz der Naturwissenschaften in der Wissenschaftskommunikation bzw. im Aufgreifen wissenschaftlicher Motive und Themen in massenmedialen Darstellungen geht vermutlich teilweise auf diese historische Entwicklung zurück.

Selbst in der Forschung zur Wissenschaftskommunikation spielen die Geisteswissenschaften eine marginale Rolle: „Fast 93\% aller Studien zu Wissenschaftskommunikation beschäftigen sich mit den so genannten MINT-Fächern, also mit Mathematik, Informatik, Natur- oder Technikwissenschaften" - Schäfer (2018) bezieht sich hier allerdings exemplarisch

4 Auch die bekannteren Titel des Genres,Wissenschaftsroman' befassen sich häufig mit naturwissen schaftlichen Themen - darunter etwa Daniel Kehlmanns "Die Vermessung der Welt" aus dem Jahr 2005 oder Frank Schätzings „Der Schwarm“ von 2004. Dieser illustriert zugleich ein zentrales Problem des Genres bzw. der Einordnung von Texten ins Genre: Die wissenschaftliche Fundierung und Reflexionstiefe in „Der Schwarm“ lässt zu wünschen übrig. Aufgrund der Nähe zur Science-Fiction wird die Abgrenzung tatsächlich genuin wissenschaftsvermittelnder Texte von jenen, die Wissenschaft eher als Folie und teilweise auch als, Mittel zum Zweck' nutzen, erschwert.

5 In seiner „Einleitung in die Geisteswissenschaften“ (1883) bemühte Dilthey sich um die Etablierung der Geisteswissenschaften als eigenständige akademische Disziplin, mit besonderem Fokus auf das "Seelenleben". 
auf den Bereich der journalistischen Medienberichterstattung sowie auf Online-Kommunikation.

In ihrem Überblicksband „Wissenschaftskommunikation - Schlüsselideen, Akteure, Fallbeispiele" (2016) betonen Weitze und Heckl schon eingangs, dass sie Wissenschaft „,im Sinne des Begriffs ,science' “ verstehen. Geistesund Sozialwissenschaften sollen damit aber „nicht ignoriert werden“, sondern statt als Gegenstand vielmehr als „Reflexionswissenschaften und theoretische Fundierung im Sinne von ,Wissenschaftskommunikationswissenschaften"' einbezogen werden (Weitze \& Heckl, 2016, S. 2). ${ }^{6}$ Auch Geier und Gottschling konstatieren sowohl in Bezug auf die Themen der Wissenschaftskommunikation als auch auf deren Erforschung eine „starke Dominanz der Naturwissenschaften" (Geier \& Gottschling, 2019, S. 283).

Die Vorstellung von Naturwissenschaften als ,hard sciences', zugespitzt ausgedrückt den ,echten', faktenbasierten Disziplinen, mag dazu geführt haben, dass naturwissenschaftliche Themen sowohl breiter in massenmedialer Bearbeitung rezipiert als auch wissenschaftsintern stärker ,beforscht' werden. ${ }^{7}$ Was sind aber weitere Aspekte? Die oben erwähnte visuelle Präsenz naturwissenschaftlicher Forschungspraktiken und -instrumente muss im Grunde schon aufmerksamkeitsökonomisch begründet sein - und damit gleichzeitig zirkulär organisiert. Viele Menschen wissen, dass Forscher:innen in Laboren bestimmte Geräte nutzen und Kittel tragen, und erkennen so beispielsweise in Werbung schnell, dass hier Wissenschaftlichkeit aufgerufen werden soll. Ihnen sind diese Motive aber zugleich nur so vertraut, weil sie in verschiedensten medialen Zusammenhängen immer wieder mit denselben Visualisierungen konfrontiert werden. Dass auch Naturwissenschaftler:innen an ihren Computern sitzen, Fachartikel verfassen und dabei genauso aussehen wie Geisteswissenschaftler:innen, ist vermutlich insbesondere für die schnelle bildliche Vermittlung von Themen kein attraktives Motiv.

Die Vermutung liegt nahe, dass das Tun derjenigen Disziplinen, bei denen das Reflexionswissen ${ }^{8}$ im Zentrum steht, insofern schwieriger zu vermitteln ist, als ihre Praktiken und Methoden visuell weniger greifbar zu sein scheinen. Vereinfacht ausgedrückt sind das etwa die (sprachliche) Interpretation ihrer Gegenstände unter Einbezug unterschiedlicher Theorien, Modelle und Methoden. Ebenso wird zwar nur ein kleiner Teil der Rezipient:innen verstehen, was genau ein:e Mathematiker:in in einem Film aufgeregt an der Tafel berechnet, aber die wissenschaftliche Praxis erscheint vertraut(er) und die grundlegende Botschaft erreicht die Zuschauer:innen. ${ }^{9}$

6 Vergleichbare Fokussierungen finden sich in unterschiedlichsten Studien, etwa auch in der psychologischen Forschung zur Wissenschaftskommunikation (vgl. dazu u.a. Bromme, 2014).

7 Eine historisch entscheidende Rolle spielt hier die Entwicklung der science studies, in deren Rahmen seit dem Beginn des 20. Jahrhunderts Methoden und Praktiken der ,modernen Naturwissenschaften' reflektiert werden. Für die Geisteswissenschaften gibt es kein vergleichbares Äquivalent und lediglich punktuelle Untersuchungen mit beispielsweise wissenssoziologischer Perspektive (vgl. Krause, Pethes \& Krause, 2017, S. 74).

8 Peter-André Alt verwendet in „Die Verheißungen der Philologie“ (2007) den Begriff des Reflexionswissens, das er spezifisch für die Philologien als prozessual versteht und damit nicht als primär "faktenbezogen" (Alt, 2007, S. 13). Die Besonderheiten der philologischen Auseinandersetzung seien etwa die Varianten und Entwicklungen, die sie aus den sprachlich erzeugten „Realitätsversionen“ herausarbeiten könne. Geprägt wurde der Begriff ursprünglich von Habermas (1973) und bezieht sich auf die Selbstreflexion von Wissenschaft.

9 Eine Historikerin im Archiv könnte als Pendant ebenfalls noch ,funktionieren'. Die Geschichtswissen- 
Reflexion zu ,zeigen' ist also sowohl für die Natur- als auch für di Geisteswissenschaften schwierig. In den Naturwissenschaften ist das für die Vermittlung aber insofern weniger hinderlich, als bei Public Science-Aktivitäten, Kinderuni oder auch in Videos Versuche, Experimente und ähnliches vorgeführt werden können. Sie ,funktionieren' visuell und beeindrucken das Publikum zum einen mit leuchtenden Farben oder chemischen Reaktionen, führen zum anderen aber auch tatsächliche Phänomene vor. Den Geisteswissenschaften fehlen diese vor allem über Visualität vermittelbaren Elemente - bzw. liegen diese ihnen ferner.

Die logische Konsequenz ist eine medial deutlich geringere Sichtbarkeit der Geisteswissenschaften. ${ }^{10}$ Dies zeigen auch die wenigen Studien zur medialen Repräsentation der Geisteswissenschaften in Bezug auf geisteswissenschaftliche Themen (Schäfer, 2018; 2018; Scheu \& Volpers, 2017). Jedoch kommen sie auch zu dem Ergebnis, dass sozial- und geisteswissenschaftliche „Akteure und Forschungsergebnisse [...] häufiger außerhalb spezialisierter Wissenschaftsressorts thematisiert und darüber hinaus auch eher mit journalistischen Formaten wie Interviews oder Kommentaren in Verbindung gebracht" (Scheu \& Volpers, 2017, S. 393) werden. Dies deckt sich mit dem Bild der Geisteswissenschaften, die die ,harten Wissenschaften' flankieren, reflektieren und kommentieren. Obwohl Geisteswissenschaftler:innen in dieser Rolle also durchaus medial präsent sind, bleiben sie und ihre Forschungspraktiken gerade aufgrund dieser Schwerpunktsetzung wenig sichtbar für die außerwissenschaftliche Öffentlichkeit.

Hier setzen die nachfolgenden Überlegungen an. Die seltenere Fokussierung auf die Geisteswissenschaften in etwa Studien der Wissenschaftskommunikationsforschung mag in Teilen auch auf die fehlende Tradition eines Äquivalents zu den science/laboratory studies zurückgehen. Vielversprechend ist aufgrund dessen der practice turn, also die aus den science studies übernommene praxeologische Perspektive auf Geisteswissenschaften - und hier speziell auf Sprach- und Literaturwissenschaften (vgl. Albrecht, Danneberg, Krämer \& Spoerhase, 2015, S. 9). Zum einen wurde in den letzten Jahren die Literaturwissenschaft als Disziplin verstärkt aus einer praxeologischen Perspektive betrachtet (vgl. dazu u.a. Martus \& Spoerhase, 2009; Albrecht et al., 2015). Zum anderen wirkt mein eigener fachlicher Hintergrund als Literaturwissenschaftlerin selbstverständlich auf die Schwerpunktsetzung ein.

Die These ist, dass die praxeologische Perspektive einen genaueren Blick auf geisteswissenschaftliche Forschungspraktiken und damit in einem nächsten Schritt erste Antworten auf die Frage ermöglichen kann, ob die geringe Sichtbarkeit der Geisteswissenschaften auf spezifische Merkmale dieser Forschungspraktiken zurückzuführen ist - und inwiefern dies schließlich Fragen der Wissenschaftsvermittlung beeinflusst.

schaften haben hier eine gewisse Sonderrolle, da sie sich aufgrund ihrer Themen, der ,Tradition' der Geschichtsvermittlung/Public History und Disziplinen wie etwa der Archäologie besser für massenmediale Visualisierungen eignen.

10 Diese wirkt sich möglicherweise auch auf das Interesse an den Geistes- und Sozialwissenschaften aus. Im Wissenschaftsbarometer 2017 (Wissenschaft im Dialog 2017) verzeichneten diese jedenfalls bei der Frage "Wie groß ist Ihr Interesse an...?" den niedrigsten Wert im Skalenbereich des ,großen Interesses'. 


\section{Doing discipline und geisteswissenschaftliche Praktiken}

Das oben angeführte „fachliche Tun als doing discipline“ (Kaufmann et al., 2019, S. 4, Herv.i.O.) spielt für meine vorliegenden Überlegungen eine zentrale Rolle. Das ,Doing' der Disziplinen wird maßgeblich durch Praktiken bestimmt - Praktiken des Forschens, des Publizierens, des Vermittelns etc. Der Blick auf Forschungspraktiken, und damit auf die Mikroebene, ist essentiell, wenn man mehr darüber erfahren möchte, was Wissenschaftler:innen tatsächlich tun. Auf dieser Basis kann schließlich weiterführend betrachtet werden, welche Möglichkeiten und Schwierigkeiten sich für die Vermittlung dieser Praktiken ergeben.

Als Praktiken des „Wissensgewinns und der Wissensvermittlung“ bestimmen Behrs, Gittel \& Klausnitzer (2013) die folgende Auswahl: beobachten, aufzeichnen, speichern; problematisieren, kombinieren, variieren; prüfen, schreiben, publizieren; rezipieren, diskutieren, polemisieren (Behrs et al., 2013, S. 5, 84) ${ }^{11}$ Diese Praktiken treffen erst einmal auf alle Wissenschaften zu. Auch Huber (2019) konstatiert Überlappungen und Berührungspunkte von etwa Hermeneutik, die eher den Geisteswissenschaften zugeschrieben werde, aber auch in anderen Disziplinen zu finden sei, oder begrifflich-theoretischer Forschung, die wiederum eher in anderen Disziplinen maßgeblich sei, sich aber ebenfalls in den Geisteswissenschaften finde. Zugleich hebt er die Bedeutung des hermeneutisch-interpretierenden Forschens für die Geisteswissenschaften nochmals hervor (Huber, 2019, S. 25). Diese Art der Forschung sei konstitutiv für Disziplinen wie etwa die Philosophie, die Kunstgeschichte, die Literaturwissenschaft - mit der Gemeinsamkeit, dass „Probleme nie abschließend gelöst, bisherige Problemlösungen immer wieder umstritten sind und das gemeinsame Suchen nach Antworten, das Selbst-Philosophieren, die bestimmende Bewegung ist" (ebd., S. 26). Wie sieht aber hermeneutischinterpretierendes Forschen aus, auch hier wieder mit Blick auf die Sicht- und ,Zeigbarkeit' gefragt?

Erste Antworten und Beispiele finden sich bei Albrecht et al. (2015), bezogen auf die Literaturwissenschaft, aber auch übertragbar auf unterschiedliche Disziplinen:

„Praktiken sind in diesem Verständnis routineförmige Tätigkeiten, die oftmals nicht vollständig durch explizierbare Regeln oder Methoden bestimmt sind, sondern in hohem Maße auf implizitem Wissen und Können - auf einem Know-how - beruhen, das durch Imitation und Beispiele erworben wird. [...] Dazu gehören etwa das Finden von Fragestellungen und die Auswahl relevanter Textstellen, die Einschätzung der Begründungsbedürftigkeit einzelner Deutungsschritte, der zitierende und argumentierende Umgang mit der Forschung, die Disposition, die rhetorische Gestaltung und das ,Aufschreiben' des Interpretationstextes." (Albrecht et al., 2015, S. 2).

11 Steffen Martus bezieht sich in seinen Ausführungen zur praxeologischen Perspektive auf die "Literatur/Wissenschaft" ebenfalls auf die Systematisierung von Behrs et al. (2013), bezeichnet die aufgelisteten Begriffe aber als „Praktiken des Wissenstransfers" (Martus, 2015, S. 191). 
Das „,Finden von Fragestellungen“ klingt zunächst präziser und fassbarer als die diffuse Vorstellung von Reflexionswissen bzw. -fähigkeit. Wer allerdings selbst regelmäßig mit der Herausforderung, Forschungsfragen einzugrenzen und je nach Ziel und Umfang anzupassen, befasst ist, ist sich der Bedeutung des impliziten Wissens, das oft nicht kleinteilig nachvollzieh- und explizierbar ist, sicherlich bewusst. Implizites Wissen spielt eine zentrale Rolle für die Praxistheorie (siehe hierzu z.B. Reckwitz, 2003) und ist entscheidender Bestandteil sozialer Praktiken - in den grundlegenden Texten dazu vor allem bezogen auf körperliche Handlungen und Routinen. Dieses teilweise kaum noch bewusste Praxiswissen kann jedoch auf unterschiedlichste Bereiche und Situationen übertragen werden, wie in diesem Fall auf das ,Betreiben' von Wissenschaft.

In diversen Ratgebern zum Verfassen schriftlicher Hausarbeiten oder der Dissertation finden sich Hinweise dazu, wie man eine bearbeitbare und relevante Fragestellung entwickelt. Dazu gehören Ratschläge wie: das zu untersuchende theoretische Konstrukt muss deutlich werden, die Forschungsfrage muss so gestellt werden, dass komplex und gehaltvoll darauf geantwortet werden kann, oder die Zeiträume sollten sinnvoll eingegrenzt werden. Solche Parameter sind sinnvoll und hilfreich, aber in letzter Konsequenz ist der Prozess nicht ,vollständig explizierbar', zu Teilen immer auch kontingent und beruht in seiner Entwicklung auf einem komplexen - und individuellen - Zusammenspiel von Erfahrung, Beispielen, situativem Kontext und vielem mehr. „Statt zu Fragen [sic], welches Wissen eine Gruppe von Personen, d.h. eine Addition von Individuen, ,besitzt', lautet die Frage, welches Wissen in einer bestimmten sozialen Praktik zum Einsatz kommt" (Reckwitz, 2003, S. 292).

Es geht also nicht um ,das Wissen', etwa im vorliegenden Beispiel um das Wissen einer gesamten Disziplin, das unbeweglich existiert und das Wissenschaftler:innen bewusst erlernen, um es anschließend anzuwenden. Geisteswissenschaftliche Forschungsergebnisse und Reflexionen entstehen im Diskurs, sind - wie oben zitiert - beweglich, offen, unabgeschlossen und werden immer wieder neu ausgehandelt. Albrecht et al. (2015) fragen in einem ähnlichen Zusammenhang:

„Inwiefern lassen sich die Voraussetzungen und Implikationen dieser Praktiken überhaupt explizit machen? Interpretations- und Argumentationsverfahren, etwa die der symptomatischen Lektüre, sind oftmals hochkomplex, ohne dass die Praktizierenden diese Komplexität unbedingt durchschauen müssten. Welche Lese- und Interpretationspraktiken liegen dem zugrunde?" (Albrecht et al., 2015, S. 13)

Dass die Praktizierenden selbst teilweise nicht kleinschrittig aufschlüsseln könnten, was sie tun, wenn sie forschen, ist insbesondere für die Vermittlung von Forschungspraktiken eine Herausforderung. Dennoch ebnet dies auch einer Multiperspektivität und Pluralität den Weg, die allzu normative Vorstellungen und entsprechend unrealistische Erwartungen minimieren können. Laut Wissenschaftsbarometer 2019 würden 64 Prozent der Befragten 
„gerne einmal erleben, wie Wissenschaftler arbeiten“ („Wissenschaft im Dia$\log /$ Kantar Emnid, 2019, Wissenschaftsbarometer 2019, Online-Publikation", о. J.). Forschungsprozesse offenzulegen und andere daran teilhaben zu lassen, ist ein wichtiger Schritt für die Wissenschaftskommunikation.

In Bezug auf die Sichtbarmachung und Vermittlung literaturwissenschaftlicher Praktiken betonen Albrecht et al., wie grundlegend die Wissensproduktion dieses Faches auf ,, sprachliche Manifestationen “ angewiesen ist: „Darstellungsverfahren zählen daher zum Kern des literaturwissenschaftlichen Praxisrepertoires." (Albrecht et al., 2015, S. 15).

Der Blick ließe sich hier noch erweitern, denn Darstellungsverfahren sind auch für andere geisteswissenschaftliche Disziplinen (und letztlich für jede Wissenschaft) elementar. Tatsächlich sind aber die Gegenstände der Literaturwissenschaft im Grunde immer Darstellungsverfahren - wie ist etwas erzählt, perspektiviert, auslegbar etc. Eben diese ,doppelte' Fokussierung auf Darstellungsverfahren macht die Konzentration auf literaturwissenschaftliche Praktiken für den vorliegenden Artikel ergiebig - nicht zuletzt, da „,über den ,modus operandi' der Literaturwissenschaft, die gewöhnlichen Verhaltensroutinen, die inkorporierten Textumgangsweisen und informellen Arbeitszusammenhänge, noch überwiegend Unklarheit herrscht", wie Schruhl (2018) konstatiert. Ein Blick in die ,Black Box' der literaturwissenschaftlichen Forschungs- und Darstellungspraktiken ist also lohnenswert. ${ }^{12}$

Ein weiteres entscheidendes Argument ist mein eigener fachlicher Hintergrund. Ich bin ausgebildete Literaturwissenschaftlerin, weswegen mir eine entsprechende Analyse zunächst näherliegt als beispielsweise die Analyse philosophischer Forschungspraktiken. Ich verfüge selbst über implizites Wissen im praxistheoretischen Sinn und bin in dieser Disziplin nicht nur Beobachterin, sondern auch Akteurin, die an den Praktiken der Disziplin partizipiert. Diesen Wissensvorteil kann ich für die Analyse fruchtbar machen, mit dem Ziel, implizite Wissensbestände explizit(er) zu machen. Damit drängt sich gleichzeitig die Frage auf, ob man als Fachfremde:r beim Hören von Podcasts überhaupt geisteswissenschaftliche Praktiken erkennen kann und ich meine hier aufgeworfene These somit selbst wieder entkräfte. Kommt man an dieser Stelle allerdings auf die science studies zurück, findet man genau diese Konstellation vor - die (vornehmliche) Beobachtung und Reflexion der Naturwissenschaften auf Basis geistes- und sozialwissenschaftlicher Methoden und Theorien (vgl. dazu etwa den Zweig der laboratory studies, maßgeblich Latour \& Woolgar (1987) und Knorr-Cetina (1981) und 1995. Die Beobachtung z.B. chemischer Experimentierpraktiken ,im Feld' durch Geistes- und Sozialwissenschaftler:innen lässt sich nicht analog auf die Praxis der Geisteswissenschaften übertragen. Es ist zu vermuten, dass hier ebenfalls das schon mehrfach angeführte geringere Potential für die Visualisierung von Forschungspraktiken einer der Gründe ist, aus denen sich keine analoge Tradition der ,humanities and social sciences studies' entwickelt hat. Darüber hinaus ist die Fremdbeobachtung schwierig, da die Geisteswis-

12 Zur praxeologischen Perspektive auf die literaturwissenschaftliche Disziplin siehe etwa Martus \& Spoerhase (2009), Martus (2015) oder Spoerhase (2015). 
senschaften (als ,Reflexionswissenschaften') schließlich die Methoden und Theorien für die Reflexion der Wissenschaften stellen - sie müssen sich also selbst beobachten und reflektieren.

Hier lässt sich, so meine These, weiteres Potential für das Format des Podcasts in den Geisteswissenschaften identifizieren, und zwar für den Bereich der Wissenschaftsforschung: Neben der Kommunikation und Vermittlung geisteswissenschaftlicher Forschungsergebnisse, -methoden und -prozesse, wird durch das Sprechen über das eigene Tun, dessen Reflexion im Moment des Sprechens sowie die gleichzeitige Vorführung der Praxis in actu mit dem auditiven Raum des Podcasts eine Art Bühne bzw. Labor der Geisteswissenschaften geschaffen. Diesen hörbaren Denkraum zu reflektieren und zu analysieren bietet meines Erachtens großes Potential für die Wissenschaftsforschung und spezifisch für eine Art kleines Analogon zu den etablierten science studies.

Anregend für diese Überlegungen scheint mir Moritz Klenks Konzept des „sprechenden Denkens" ${ }^{\text {"13 }}$ zu sein:

„Das Zeigen selbst kann nicht gezeigt werden, sondern zeigt sich in der Durchführung. Das sprechende Denken, um das es hier geht, ist als Denken Vermittlung von Gegenstand und Reflexion. Als diese aber und als Sprechen ist es darin die Vermittlung von Durchführung und Darstellung. Genauer: es ist Darstellung als Durchführung." (Klenk, 2020, S. 9, Herv.i.O.)

Klenk bestimmt das Denken hier zugleich als „einfache Praxis“, als einen Prozess, der ,durchgeführt' wird, und als „Weise der Darstellung” (ebd., Herv.i.O.). Diese Gleichzeitigkeit von Reflexion und „Darstellung “ prägt auch die vorliegenden Ausführungen. Mit Blick auf die Wissenschaftsforschung beklagt Klenk die ausgebliebene Reflexion des Sprechens, trotz der von jenem stark geprägten Wissenschaftspraxis (vgl. Klenk, 2020, S. 153f). In seinem Dissertationsprojekt, das einer ,experimentellen Kulturwissenschaft' verpflichtet ist, ist das Sprechen demgemäß zentral. Die Arbeit baut auf einem Podcast-Experiment auf, für das Klenk im Jahr 2017365 Folgen, also eine Episode täglich, aufgenommen hat, in denen er sich (im Selbstgespräch) mit seiner Forschung befasst, Texte diskutiert, Überlegungen formuliert, reflektiert und verknüpft. ${ }^{14}$

Seine dialektische Bestimmung des Hörens und des Sprechens („Sprechen ist immer ein Hören, ein hörendes Mitverstehen des Gesprochenen“, Klenk (2020), 167) belegt einmal mehr das Potential des Podcast-Formats und unterscheidet es etwa von einer Audioaufnahme, die man für sich selbst anfertigt und speichert. Das Selbstgespräch kann sicherlich auch in dieser Variante Erkenntnisse generieren und Raum für sprachliche Reflexionen bieten. Ein Podcast ist aber in seiner Anlage bereits auf soziale Interaktion ausgerichtet und somit quasi ein idealtypischer Modus des sprechenden Denkens.

13 Für den Hinweis auf Moritz Klenk und sein Konzept des „sprechenden Denkens“ gilt mein Dank den Herausgeber:innen.

14 Podlog. Das Podcast-Logbuch: https://podlog.noradio.eu (Zugriff am 03.09.2020). 
Interaktion zum einen mit Blick auf die Gestaltung des Podcasts selbst, wenn es Interview- oder Gesprächspodcasts sind, in denen quasi primär interagiert wird. Zum anderen schaffen Podcasts durch ihre öffentliche Verfügbarkeit immer auch eine Art sekundäre Interaktionsebene, durch die grundsätzliche Möglichkeit ihrer Rezeption und Auseinandersetzung mit ihnen.

Hierbei geht es nicht darum, und damit komme ich zurück auf die Frage, ob Fachfremde generell geisteswissenschaftliche Praktiken erkennen können, dass Lai:innen explizite Praktiken als eben diese identifizieren können und anschließend verstanden haben, wie die Forschung einer bestimmten Disziplin funktioniert und organisiert ist. Die Idee ist vielmehr, dass über das ,Doing' die Vielfalt und Vorläufigkeit der Auseinandersetzung mit Gegenständen erlebbar und deutlich wird, ebenso wie auch Teile impliziten Wissens doch dazu nachfolgend mehr.

\section{Vom ,Verfertigen der Gedanken': Podcasts in der geisteswissenschaftlichen Wissenschaftskommunikation}

Meine These ist, dass die Wissensvermittlung über das Format der Wissenschaftspodcasts besonders viel Potential für die Geisteswissenschaften birgt. Sie bieten erstens ausreichend Raum: Podcasts dürfen sehr lang sein, etwa im Unterschied zu exakt getimten und konzisen Radiobeiträgen. Sie ermöglichen außerdem zweitens, auf verschiedenen Ebenen zu zeigen, was geisteswissenschaftliche Forschung ausmacht.

Neben der gegebenen Breite und Ausformulierung von Gedanken wird - je nach Schnittstil in der Postproduktion, sei einschränkend erwähnt - auch die ,Verfertigung der Gedanken' dokumentiert; so etwa die unterschiedlichen Sprechgeschwindigkeiten, Pausen oder das Ins-Stocken-Geraten der Sprechenden. In Wissenschaftspodcasts bedeutet das etwa ein Nebeneinander von sehr schnellen und sicher vorgetragenen Passagen, die vermutlich schon häufiger von der Sprecherin oder vom Sprecher durchdacht und beschrieben wurden, und von spontaneren, noch , unfertigeren' Überlegungen. Diese werden oft vorsichtiger formuliert, gleichen Suchbewegungen, bei denen neu angesetzt wird und herausgehört werden kann, an welchem Punkt in der Forschung bzw. der Reflexion die Person steht. Dies ist bereits eine der möglichen Ebenen, auf denen man sich geisteswissenschaftlichen Forschungspraktiken nähern kann, die Suchbewegungen und zirkulären Interpretationsprozessen gleichen können.

„It not only reflects its own research question/s, but reflects the reflection itself: the process of knowledge-production, the presentation and representation of language and voice, the narrative and dramaturgy, and the aesthetics of sound." (Groth \& Samson, 2016, o.S.)

Dieser Auszug aus dem Manifest zu Audio Papers ${ }^{15}$ betont die ,Meta-

15 Audio Papers werden im besagten Manifest als ,neues Artikelformat' eingeführt: „Audio papers resemble the regular essay or the academic text in that they deal with a certain topic of interest, but 
Reflexion', die die Audioform durch Elemente wie Stimme und Sound ausmacht und um die es hier ebenfalls geht. Der Forschungsprozess erscheint dadurch noch beweglicher, was besonderes Potential birgt, um die Vorläufigkeit wissenschaftlicher Ergebnisse vermitteln zu können.

Zusätzlich zur inhaltlichen Ebene, also dem Thema, um das es im Podcast geht und über das man Informationen zu geisteswissenschaftlichen Fragen gewinnt, ist die Metaebene aufschlussreich, die geisteswissenschaftliche Praktiken im Moment des ,Doing' hörbar werden lässt: Wie geht die Person mit bestimmten Fragen um, welche Strategien wendet sie an, um auf Themen zu reagieren, wie systematisiert sie ihre Gedanken?

Carlos Spoerhase schreibt über das Seminar als Labor der Philologie ${ }^{16}$, dass dort „nicht das Vermitteln von Faktenwissen oder Regelkenntnissen im Vordergrund steht, sondern das wiederholte Einüben von Fertigkeiten und Fähigkeiten im gemeinsamen praktischen Umgang mit disziplinspezifischen ,Artefakten' und ,Instrumenten' " (Spoerhase, 2015, S. 62). Dies lässt sich auf den Raum des Podcasts übertragen, mit der Erweiterung um das partizipative Moment der öffentlich (und somit auch außerakademisch) zugänglichen Form. In der Interaktion von (in diesem Fall) Geisteswissenschaftler:innen können diese Fertigkeiten sowohl im ,Doing' erlebt als auch von den Sprecher:innen selbst weiter eingeübt oder gar hinterfragt werden. Im Folgenden möchte ich dies an zwei Beispielen schlaglichtartig beschreiben. Dabei nähere ich mich den jeweiligen Gesprächen von (Literatur-)Wissenschaftler:innen zunächst beobachtend und explorativ, aus praxeologischer Perspektive sowie vor dem Hintergrund der science studies. Das Ziel ist dabei, einerseits exemplarisch inhaltliche Informationen zu literaturwissenschaftlichen Praktiken zu erhalten, andererseits die Praktiken, wie bereits beschrieben, in actu , anhören'/erleben zu können.

\subsection{Exemplarische Analysen geisteswissenschaftlicher Wissenschaftspodcasts}

\subsubsection{Das Neue Berlin}

Aus der Selbstbeschreibung des Podcasts Das Neue Berlin erfahren wir, worum es den beiden Produzenten geht:

„Wir diskutieren über das, was uns interessiert: Wie funktioniert Gesellschaft? Was heißt es heute, politisch zu sein? Welches Wissen ordnet unsere Welt? ,Feuilleton von unten' nennen wir das. Das heißt für uns nicht Kulturrundschau und Rezension - Feuilleton verstehen wir als ein öffentliches Nachdenken über Gesellschaft im weitesten

presented in the form of an audio production. The audio paper is an extension of the written paper through its specific use of media, a sonic awareness of aesthetics and materiality, and creative approach towards communication." (Groth \& Samson, 2016) https://seismograf.org/fokus/fluidsounds/audio paper manifesto. Vgl. dazu ferner das online bereitgestellte "Gespräch über Audio Paper" zwischen Stephan Porombka und Holger Schulze, das auch als Abdruck erschienen ist (vgl. Porombka \& Schulze, 2018).

16 Dies ist einer der wenigen Versuche, die Laborsituation aus den science studies auf die Geisteswissenschaften zu übertragen und für diese fruchtbar zu machen. 
Sinne. Dabei versuchen wir, Perspektiven aus den Geistes- und Sozialwissenschaften zeitdiagnostisch produktiv zu machen." (Das Neue Berlin, https://dasneue.berlin/ueber-uns/)

Dieses Ziel verfolgen sie entweder in Gesprächen untereinander oder im Austausch mit Gästen. Hier wird eine wichtige Funktion des Formats Podcast deutlich - es soll in diesem Fall geistes- und sozialwissenschaftliche Perspektiven "zeitdiagnostisch produktiv" machen und dadurch aktuelle gesellschaftliche Fragen wissenschaftlich betrachten - im Dialog. Mit der Formulierung des "Feuilletons von unten" wird eine unabhängige, aber wissenschaftlich informierte Perspektive auf gesellschaftliche Entwicklungen und Phänomene angestrebt.

Tabelle 1: Podcast-Steckbrief Das Neue Berlin. Quelle: eigene Darstellung.

\begin{tabular}{ll}
\hline Hosts | Hintergrund & Jan Wetzel, Leo Schwarz; privat betrieben \\
\hline Disziplinäre Perspektive & Geistes- und Sozialwissenschaften \\
Zeitraum | Episoden & Episode 1: 22.03.2018, aktuelle Episode 56: 17.08.2020 \\
& insgesamt 56 Episoden \\
Episodenlänge & zwischen ca. 46 und 156 Minuten ( $\emptyset$ ca. 100 Minuten) \\
Gesprächsformat & Gespräch zwischen den Hosts oder mit Gästen, in jüngerer Zeit vor \\
& allem mit Gästen \\
Link zum Podcast & https://dasneue.berlin \\
\hline
\end{tabular}

Für die nachfolgende Analyse habe ich die erste Folge „Wen kümmert' wer spricht? Reden übers Reden mit Heinrich von Kleist“"17 (Berlin, 2018) ausgewählt, in der die Sprecher sich zu zweit einem Text von Heinrich von Kleist nähern. Die Folge ist eher im weiteren Sinne literaturwissenschaftlich, da Jan Wetzel, einer der Produzenten, Soziologe ist, und Leo Schwarz, die andere Hälfte des Neuen Berlins, Philosophie, Germanistik, Kultur- und Sozialwissenschaften studiert hat. In der Episode gibt es also interdisziplinäre Zugänge, sie lässt sich aber aufgrund der Art der Annäherung an den Text durchaus als literaturwissenschaftlich (und hermeneutisch) charakterisieren. Darüber hinaus lässt sich der Kleist-Text nahezu sinnbildlich für den oben beschriebenen Prozess des Denkens, Abwägens, Sortierens, Priorisierens in Podcasts verstehen und bietet mit dem Thema der ,allmählichen Verfertigung der Gedanken beim Reden' sowohl inhaltlich als auch in Bezug auf die Metaebene einen idealen Rahmen. Vor dem Hintergrund von Klenks "sprechendem Denken" müsste zwar das in der Verfertigung implizierte ,Fertigwerden' relativiert werden, um dem Prozesshaften, Zirkulären und Unabgeschlossenen Rechnung zu tragen, die „Darstellung als Durchführung“ lässt sich aber meines Erachtens dennoch mit dem Prozess der Verfertigung zusammendenken.

Nach der Begrüßung und Vorrede stellen die Sprecher zunächst kurz den Text vor und steigen anschließend mit einem Zitat zu Beginn des Textes ein, in dem Kleist passenderweise dazu rät, sich mit jemandem auszutauschen, wenn man etwas wissen wolle. Mehr oder weniger nach dem Prinzip des close rea-

17 https://dasneue.berlin/2018/03/22/dnb1-allmaehliches-verfertigen/ (Zugriff am 01.03.2020). 
ding ${ }^{18}$ diskutieren sie verschiedene Begriffe und Formulierungen im Satz, ordnen ihre Bedeutung in den zeitlichen Kontext ein, assoziieren aber auch freier und setzen sie mit ,zeitgenössischen' Vorstellungen in Beziehung. Daran lassen sich strukturierende Praktiken der (literatur-)wissenschaftlichen Forschung ablesen (vielmehr: abhören), also etwa eine semantische Einordnung, aber auch mögliche literaturgeschichtliche Hinweise - was sie fast schon spöttisch tun, in dem sie ein „literaturwissenschaftliches Klischee“ (Berlin, 2018, S. 00:12:03) aufrufen, dem zufolge Kleist bekannt sei für „die besten literarischen Nebensätze“, „aber das nur so am Rande” (ebd., 00:12:13).

Beim nächsten Kleist-Zitat werden dann interpretierende und Transferpraktiken deutlicher. Zur besseren Orientierung zunächst das entsprechende Zitat:

„Aber weil ich doch irgend eine dunkle Vorstellung habe, die mit dem, was ich suche, von fern her in einiger Verbindung steht, so prägt, wenn ich nur dreist damit den Anfang mache, das Gemüt, während die Rede fortschreitet, in der Notwendigkeit, dem Anfang nun auch ein Ende zu finden, jene verworrene Vorstellung zur völligen Deutlichkeit aus, dergestalt, daß die Erkenntnis, zu meinem Erstaunen, mit der Periode fertig ist." (Kleist, o. J., S. 319f)

Die „dunkle Vorstellung“ wird von einem der Sprecher als das Gefühl eines Gedankens, der aber noch nicht formuliert werden kann, ,übersetzt', die ,fortschreitende Rede' aufgrund des Zugeständnisses, die Rede nicht unter Kontrolle zu haben, als Entfremdungserfahrung interpretiert (Berlin, 2018, S. 00:13:51). Sein Gesprächspartner stimmt zu und überträgt die Kleist'schen Worte auf die Gegenwart und sein eigenes Empfinden: „Ich muss auch gleich irgendwie an so Situationen denken, wo mich dann auch so leichte Panik beschleicht während des Redens, weil ich noch nicht weiß, was der nächste Satz ist" (ebd., ab 00:14:02). Hier gibt er den Zuhörer:innen noch den Tipp, über etwas anderes zu sprechen und nicht beim Thema zu bleiben, quasi bewusst den Faden zu verlieren. Wenn man sich eine Weile in akademischen Zusammenhängen aufhält, kann man auch diesen Hinweis als Praktik identifizieren, insbesondere im Rahmen von Vorträgen oder Kolloquien, in denen sich das ausweichende Antworten auf kritische Nachfragen zu einer ganz eigenen, nahezu kunstvollen Praktik entwickelt hat. Nach der sehr gezielten semantischen Analyse geht es also plötzlich um Praktiken in (dialogischen) akademischen (Gesprächs-)Situationen, die aber auch problemlos auf andere Felder und Situationen übertragen werden können.

Neben der inhaltlichen Ebene und dem, was die beiden über Kleist zu sagen haben, verfolgt man auf der Metaebene fortlaufend die zunehmende, Verfertigung der Gedanken', das „,sprechende Denken“, und die weiterführenden Überlegungen und Denkprozesse im Gespräch der beiden über den Text. So kann neben einer Vorführung geisteswissenschaftlicher Praktiken des Inter-

\footnotetext{
18 Darunter wird in der Literaturwissenschaft die präzise Auseinandersetzung mit Texten bzw. Textauszügen verstanden, die kleinste Details, Bedeutungen, sprachliche Figuren etc. berücksichtigt und nur den Text an sich in den Mittelpunkt stellt. Das close reading als Analysetechnik entstammt dem New Criticism, der von Cleanth Brooks (1971 [1947]) und Wimsatt/Beardsley (1946) ausgehend in den 1940er Jahren entwickelt wurde.
} 
pretierens, Verweisens oder Einordnens gleichermaßen ihre Reflexion, ihre Prozess- und Ereignishaftigkeit (vgl. Klenk, 2020, S. 168) verfolgt werden.

„Denn nicht wir wissen, es ist allererst ein gewisser Zustand unsrer, welcher weiß", schreibt Kleist - das würde man eigentlich auch im 21. Jahrhundert so sagen, konstatiert einer der Sprecher (Berlin, 2018, ab 00:37:41). Das Subjekt, um das es hier gehe, gebe es zwar, es sei aber nicht fix, werde immer neu hergestellt und in neue Zustände versetzt. Der andere Sprecher zieht ein Nietzsche-Zitat aus „Jenseits von Gut und Böse” heran, „um es geistesgeschichtlich noch mal richtig krachen zu lassen" (ebd., 00:39:28) und lässt dabei einen Hinweis auf eine „Kant-Schelte“ im Nietzsche-Zitat fallen. Abgesehen von der inhaltlichen Begründung dafür, dem Kleist-Text eine Nietzsche-Referenz (inklusive Kant-Verweis) an die Seite zu stellen, wird hier sehr eindrücklich die geisteswissenschaftliche Praktik vorgeführt, Literatur bzw. Theoretiker:innen zu konsultieren und den vorliegenden Text auf größere geistesgeschichtliche Fragestellungen zu übertragen - u.a. bei der Suche nach Definitionen bzw. Annäherungen an bestimmte Begriffe.

Was relativ deutlich zu erkennen ist: Die Argumentationsstruktur beider Sprecher ist der schriftlichen geisteswissenschaftlichen auch im Dialog sehr ähnlich. So werden z.B. Wechsel und die „Zielgerade“ angekündigt (ebd., 01:02:07), parallel zu Überschriften oder einer Gliederung. Die besagten Verweise auf Theorien, ,Denkschulen' oder Kontexte und geistesgeschichtliche Einordnungen folgen ,klassischer' geisteswissenschaftlicher Forschungspraxis, ebenso die kontinuierliche Arbeit mit Zitaten. Die davon abweichenden Momente sind die der Übertragung von Reflexionen auf das eigene Erleben und die Alltagsrealität, auf die Wahrnehmung der verhandelten Fragen und Gedanken „im 21. Jahrhundert", also quasi die Anwendbarkeit des Textes von Kleist und seine Aktualität in der Gegenwart. Vermeintlich schwierig zu vermittelnde geisteswissenschaftliche Forschungspraktiken lassen sich so im Format des Podcasts ,anhören' und erzählen, ohne sie aber zu erklären in ihrer Vorführung und der Möglichkeit, an ihrer ,Verfertigung' teilzuhaben (was auch im Sinne Klenks und seiner Vorstellung von Dialogik als Dialektik wäre).

Es gäbe diverse weitere Szenen, an denen man etwa sprachliche Markierungen und ihre Auswirkungen auf die Argumentationspraxis aufzeigen könnte. Es sollte aber bereits deutlich geworden sein, dass in dieser Folge des Podcasts Form und Inhalt für die vorliegende Analyse mehr oder weniger idealtypisch sind, und inwiefern hier allgemein geistes- und spezifisch literaturwissenschaftliche Forschungspraktiken nachvollzogen werden können.

\subsubsection{Praktisch Theoretisch}

Der zweite ausgewählte Wissenschaftspodcast ist Praktisch Theoretisch, den ich selbst gemeinsam mit meinem Kollegen Stephan Fasold produziere. Darin unterhalten wir uns mit Wissenschaftler:innen unterschiedlicher Disziplinen über praktische Fragen, die mit wissenschaftlichen Theorien bzw. wissen- 
schaftlich beantwortet werden sollen. Zum einen sind die Erfahrungen, die ich dabei bisher gemacht habe, Anstoß der hier ausgeführten Thesen. Zum anderen gibt es eine Folge, die sich dezidiert mit Literatur und Literaturwissenschaft auseinandersetzt und sich somit ideal für den gewählten Fokus dieses Artikels eignet.

Tabelle 2: Podcast-Steckbrief Praktisch Theoretisch. Quelle: eigene Darstellung.

\begin{tabular}{ll}
\hline Hosts | Hintergrund & $\begin{array}{l}\text { Stephan Fasold, Rebecca Moltmann; lose im Kontext des } \\
\text { Sonderforschungsbereichs „Praktiken des Vergleichens“ } \\
\text { (Universität Bielefeld), aber thematisch unabhängig }\end{array}$ \\
\hline Disziplinäre Perspektive & $\begin{array}{l}\text { Geistes- und Sozialwissenschaften } \\
\text { Episode 0: 28.04.2019, aktuelle Episode 20: 11.08.2020 } \\
\text { Zeitraum | Episoden }\end{array}$ \\
insgesamt 28 Episoden (inkl. „Außer der Reihe”-Episoden) \\
zwischen ca. 20 und 87 Minuten ( $\emptyset$ ca. 60 Minuten) \\
Gesprächsformat & Gespräch mit Gästen, seltener nur zwischen den beiden Hosts \\
Link zum Podcast & htt ps://praktischtheoretisch.podigee.io \\
\hline
\end{tabular}

Die Episode „Lernen wir aus Literatur und wozu eigentlich Literaturwissenschaft? Gespräch mit dem Literaturwissenschaftler Lutz Graner" ${ }^{\text {"19 }}$ (Theoretisch, 2019) unterscheidet sich von der Das Neue Berlin-Folge zunächst durch die Gestaltung. Dort wird ein Text gemeinsam von den beiden Produzenten besprochen, die mit Beginn der Folge direkt in die Annäherung und seine Interpretation einsteigen. In der Praktisch Theoretisch-Episode findet hingegen eine Interviewsituation mit einem Gast statt, dem Literaturwissenschaftler Lutz Graner. Dadurch, dass ich als ,Gastgeberin' ebenfalls einen literaturwissenschaftlichen Hintergrund habe, gibt es aber auch hier immer wieder eher dialogische Passagen und Diskussionen. Stephan Fasold, der selbst Historiker ist, war zum Zeitpunkt der Aufnahme auf Archivreise. Die Gesprächssituation war somit vermutlich aufgrund des gemeinsamen disziplinären Hintergrunds dialogischer als sie es etwa durch Verständnisnachfragen aus einer anderen wissenschaftlichen Perspektive gewesen wäre.

Unsere Folgen sind grundsätzlich auf ein Nebeneinander von eher zugespitzten Fragen, angelehnt an den ,praktischen Aufhänger', und von gesetzten Impulsen für dialogische Momente ausgerichtet. Wie der Name verrät, steht das Zusammenspiel von Praxis und Theorie im Fokus - Wissenstransfer, Vermittlung, Anwendbarkeit sind also Kategorien, die immer wieder eine Rolle spielen. „Praktisch Theoretisch“ ließe sich somit in Bezug auf die Produktion der explizit metareflexiven Auseinandersetzung mit den Praktiken, Methoden und Prozessen wissenschaftlicher Disziplinen zuordnen. Auch die hier analysierte Praktisch Theoretisch-Folge ist von vornherein stärker auf der metareflexiven Ebene angesiedelt, da die Frage im Zentrum steht, wie man sich literaturwissenschaftliche Arbeit vorstellen kann und wieso man sie machen wollen sollte.

Hier erfahren Zuhörer:innen also ausgestellter etwas über die Literaturwissenschaft und ihre Verfahren. Beispielsweise wird hier die Methode des close reading explizit besprochen (Theoretisch, 2019, ab 00:14:04) und als zen-

19 https://praktischtheoretisch.podigee.io/18-literatur-wissenschaft (Zugriff am 01.03.2020). 
tral für die Seminargestaltung bewertet. Dennoch gibt es immer wieder Praktiken, die im Gespräch nicht explizit benannt werden, aber entscheidend für die wissenschaftliche Forschung sind, wie etwa die Themenwahl. Wie findet man ein Thema, ob nun für ein Seminar oder eine Studie, worauf basiert die Idee und mit welchem Ziel verfolgt man sie? Hier geht es etwa um das Seminarthema der „literarischen Weltuntergänge“ (ebd., ab 00:15:06), für das Texte wie die Johannesoffenbarung oder Heinrich Heines „Götterdämmerung" gelesen werden. Im Gespräch darüber wird deutlich, dass diese sowohl per close reading und textimmanent betrachtet als auch intertextuelle Bezüge auf Basis der motivgeschichtlichen Auseinandersetzung über diverse Zeitströmungen und Textsorten hergestellt werden können. Im Dialog wird so vermittelt, welche unterschiedlichen Möglichkeiten und Potentiale die Analyse von Literatur hat. Am spezifischen Beispiel der literarischen Weltuntergänge wird sichtbar, dass sich das Motiv der zugrunde gehenden Welt schon sehr früh findet und sich bis heute konstant durch die Literatur zieht (ebd., ab 00:17:26).

Auch in dieser Folge wird die oben schon thematisierte ,Kongruenz' von besprochenen Inhalten und der Art und Weise der gesprochenen Darstellung dieser Inhalte besonders deutlich - also die Entsprechung von Inhalt und Form: „Darstellungsverfahren zählen [...] zum Kern des literaturwissenschaftlichen Praxisrepertoires", um noch einmal an die oben zitierte Aussage von Albrecht et al. (2015), 15 zu erinnern.

Ab ca. 00:24:16 (Theoretisch, 2019) bewegt sich Lutz Graner anhand mehrerer argumentativer „Schlaufen und Drehungen“ um die Frage, was Literatur, also Sprachkunstwerke, speziell macht, und führt dabei performativ die Herausforderung eben dieser Darstellungsverfahren vor, die ihrem Gegenstand gerecht werden müssen. Insbesondere im Bereich der Philologien sind die analysierenden Texte auch immer wieder selbst nahezu literarische Texte; zwar gibt es unterschiedliche Herangehensweisen und Schreibstile, dennoch lässt sich diese Beobachtung in der Forschungsliteratur relativ häufig machen.

Allein diese sprachlichen Suchbewegungen und die verschiedenen Ebenen, mit denen hier operiert wird, erfordern einen ausreichenden Raum, um "komplexe wissenschaftliche Sachverhalte in der Zeit, die es eben brauche" auszubreiten, so Tim Pritlove, ein bekannter Podcaster der, ersten Welle', im Merton-Magazin des Stifterverbands für die Deutsche Wissenschaft (Grasberger, 2016). Im selben Artikel wird auch Nicolas Wöhrl zitiert, ebenfalls einer der bekannteren Wissenschaftspodcaster, der gemeinsam mit Reinhard Remfort den Physik-Wissenschaftspodcast Methodisch inkorrekt produziert: „[I]n Podcasts lasse sich, über Scheitern als Chance zum Lernen, auch das Prozesshafte von Wissenschaft darstellen" (Grasberger, 2016).

Zwar geht es hier weniger um eine gescheiterte Versuchsreihe im Labor, aber in der Praktisch Theoretisch-Folge wird etwa das Thema des literarischen Kanons diskutiert, der nach wie vor in vielen Universitäten sehr eurozentristisch und männlich dominiert ist, und damit offensichtlich Versäumnisse in der kritischen Hinterfragung, Reflexion, Veränderung oder Erweiterung von solchen gesetzten Kategorien entlarvt, beispielsweise durch eine Thematisierung in 
Seminaren. Durch die offene Gesprächsform und die Schlaufen und vielleicht auch Abzweigungen, die in freien, nicht geskripteten Gesprächen entstehen, kann ein offenerer Raum für die kritische Auseinandersetzung mit etablierten Formen entstehen - wie in diesem Fall der problematischen Herausbildung eines Kanons, aber auch Genres, ,Epochen' etc. Gerade die dialogische Annäherung an ein Thema ermöglicht schon im Prozess des ,Verfertigens der Gedanken', die Vielfalt unterschiedlicher Perspektiven vorzuführen und gleichzeitig klarzumachen, dass diese nebeneinanderstehen können, ohne zu einer Lösung oder Entscheidung kommen zu müssen. Dies ist einer der Schritte, die Zuhörer:innen verdeutlichen können, dass es in der wissenschaftlichen Auseinandersetzung nicht um die eine Lösung, ein ,Richtig' oder ,Falsch' geht.

Gemäß dem Titel der Folge und der Frage, was man eigentlich von Literaturwissenschaft hat, formuliert Lutz Graner:

„Das heißt, das Denken zu schulen, kann man an ganz vielen Dingen praktizieren [...], und literarische Texte sind eben ganz spezielle [...] Texte und da kann man eben auf ganz besondere Art und Weise wiederum seine Denk- und Sprachfähigkeiten entwickeln, und das wäre für mich das, was das Relevante daran ist. Es geht also nicht um das Entnehmen von konkreten Wissenshorizonten aus diesen Texten [...], sondern mich an diesem Sprachkunstwerk [...] abarbeiten, zu überlegen, wie ist denn das gemeint, [...] warum ist denn das so mehrdeutig. [...] Langer Rede, kurzer Sinn: Wenn man nun diese Frage der Anwendbarkeit beantworten muss, wär das meine Antwort: Anders denken." (Theoretisch, 2019, ab 00:49:17)

Er erläutert aus seiner Perspektive weiter, dass es letztlich nicht primär um Autor:in X oder Inhalte von Romanen gehe, was Personen außerhalb der Literaturwissenschaft häufig glauben und sich deswegen fragen, welche Relevanz das für sie oder auch für aktuelle gesellschaftliche Situationen haben solle. Die Kompetenz, , anders über Welt nachzudenken“ (Theoretisch, 2019, S. 00:52:40), gehe über Literatur und ihre Gemachtheit hinaus und lasse sich auf Probleme in der Welt übertragen.

Dieses andere, durchaus individuell ausgeprägte Denken als Ergebnis eines literaturwissenschaftlichen Studiums, lässt sich in diesem Beispiel auf die spezifische Haltung des Literaturwissenschaftlers, der sie vertritt, übertragen, und zeigt erneut die Relevanz von Reflexion, Interpretation und Transfer auf. Die vielen Schleifen dieser Argumentation, die immer wieder eingeschobenen Relativsätze, die Spezifizierungen des bereits Spezifizierten sowie die Relativierungen des zuvor Behaupteten sind genuine Elemente, um in der Literaturwissenschaft zu Beobachtungen und Ergebnissen zu kommen. Man kann in dieser Folge dem Literaturwissenschaftler buchstäblich beim Denken zuhören und den Abzweigungen, Verästelungen und sprachlichen Volten des Interpretationsprozesses folgen. 


\subsection{3 „Sprechendes Denken“ und das literaturwissenschaftliche Labor}

Die beiden Podcast-Episoden einander als Beispiele für unterschiedliche Ausprägungen des Potentials geisteswissenschaftlicher Wissensvermittlung gegenüberzustellen, erscheint mir zu holzschnittartig. Insbesondere in Bezug auf die Vorstellung des „sprechenden Denkens" sowie die Gleichzeitigkeit von ,Sprechen über' und „Darstellung als Durchführung“ sollte deutlich geworden sein, dass in beiden ausgewählten Folgen entsprechende Passagen ,heraushörbar' sind. Diese beziehen sich etwa auf das wissenschaftliche System mit seinen Routinen, impliziten Praktiken und Eigenheiten, dem die Sprecher:innen angehören. Teilweise wird dieses explizit ironisch kommentiert, etwa durch Verweise auf akademische Gepflogenheiten wie das gezielte Ablenken von der eigentlichen Frage, sollte man auf diese keine informierte Antwort geben können. Praktiken dieser Art können die Zuhörer:innen aber auch in actu erleben, da schließlich alle Sprecher:innen innerhalb des besagten Systems ,fachlich sozialisiert' wurden und sie, Selbstreflexion hin oder her, schlicht internalisiert haben.

Ähnliches gilt sowohl für die inhaltlich-thematische als auch für die metareflexive Ebene der Gespräche. Literaturwissenschaftliche Praktiken oder Bündel von Praktiken ,,sind oftmals hochkomplex, ohne dass die Praktizierenden diese Komplexität unbedingt durchschauen müssten" (Albrecht et al., 2015, S. 13). Die zirkuläre Bewegung des „,sprechenden Denkens" erstreckt sich auch auf diese Prozesse und bietet eine Möglichkeit, Unauflösbarkeiten ,auszuhalten', sich ihnen reflexiv zu nähern, statt sie in kleinste Einzelheiten zu zerlegen und damit erklären zu wollen.

Dies im Format des Podcasts darzustellen, durchzuführen und zu reflektieren, ist meines Erachtens insbesondere für die Geisteswissenschaften vielversprechend - zum einen für die Vermittlung ihrer Praktiken und ihre Sichtbarkeit, zum anderen für die Weiterentwicklung ihrer Selbstreflexion und damit ihrer Position als Gegenstand der Wissenschaftsforschung. Die Analysen sollten hierzu aufzeigen, inwiefern der auditive Raum des Podcasts mit dem „sprechenden Denken" etwa zu einer Art literaturwissenschaftlichem Labor werden kann.

\section{Ausblick: Wissenstransfer und Partizipation}

Zum Abschluss möchte ich auf einige entscheidende Aspekte des Potentials von Podcasts für die Wissenschaftskommunikation eingehen, und zwar zu Wissenstransfer und Partizipation an Wissen. Die Forschungsliteratur zu Podcasts ist, insbesondere im deutschsprachigen Raum und zum Themenkomplex der Wissenschaftspodcasts, noch sehr ausbaufähig. Es gibt zwar einige Studien zum didaktischen Einsatz von Podcasts in der Lehre und ihrem Nutzen im Bildungsbereich ${ }^{20}$, um diesen spezifischen Fokus geht es mir in

20 Vgl. hierzu die Literatursammlung „Researching Podcasts/Podcasting“ von Nele Heise und dort vor allem die Kategorie ,Podcasting, (Higher) Education \& Teaching' (verfügbar unter https://docs.google. com/document/d/1PCqveZwFSexeMshl1rxBW6VbpCrC_VyJddyTwLifOR4; Zugriff am 03.09.2020). 
meiner Argumentation jedoch nicht.

Wenn ich hier von Partizipation und Wissenstransfer spreche, beziehe ich mich also nicht auf diesen pädagogischen Hintergrund. Mir geht es um das ,Hineinhören in geisteswissenschaftliche Forschungspraktiken', die Teilhabe am ,Doing' der Wissenschaftler:innen und die möglichen Erkenntnisse, die Zuhörer:innen gewinnen können - von konkreten inhaltlichen Aspekten bis zum Moment des Miterlebens, wie Wissenschaftler:innen arbeiten, ihre Gedanken sortieren und formulieren. Gerade für die Debatte über die Glaubwürdigkeit von Wissenschaft, die in den letzten Jahren wieder vermehrt um Begriffe wie ,alternative Fakten' oder ,postfaktisches Zeitalter' geführt wurde (siehe dazu etwa Lepore 2016 oder Jacobsen 2016), birgt dieses Zuhören meines Erachtens besonderes Potential. Es kann nicht oft genug thematisiert werden, dass es nicht um absolute Erklärungen und das Markieren von ,richtig' und ,falsch' geht, sondern um den Weg des Erschließens und des prozessualen Wissens.

Die häufig geforderte Transparenz und Konzentration auf den Forschungsprozess scheinen nach wie vor eher selten im Fokus vieler Wissenschaftskommunikationsmaßnahmen zu stehen. Das Format des Podcasts bietet hier nicht nur Möglichkeiten für die Wissensvermittlung an ein außerakademisches $\mathrm{Pu}$ blikum, sondern kann selbst Instrument wissenschaftlicher Praxis sein und somit die Wissensproduktion mitgestalten. ${ }^{21}$

Zum Wissensbegriff und seiner Realisierung schreiben Behrs et al.:

„Als Ergebnis von Zeichenordnungen und sozialen Aushandlungsprozessen, formgebenden Regularien und autorisierenden Techniken realisiert sich Wissen vielmehr stets in Prozessen kommunikativen Austauschs. Daher sind Vorgänge der Aufzeichnung und Speicherung, der Instruktion und Einübung, der Adaptation und Modifikation nicht periphere und zu vernachlässigende Begleitphänome [sic] der Produktion, reiner' Erkenntnis, sondern konstitutive Funktionselemente eines Wissens, das der stetigen Weitergabe und Aktualisierung bedarf, um als Wissen aufgenommen und wirken zu können." (Behrs et al., 2013, S. 13, Herv.i.O.)

Den Autoren geht es hier zwar vor allem um interne Konzepte und Theoriebildungen innerhalb von Wissenschaftskulturen, aber gerade diese Entwicklungen und Praktiken können in einem Podcast durch Gespräche zwischen Wissenschaftler:innen abgebildet und vorgeführt werden, auch ohne als Zuhörer:in alle Begriffe, das Kontextwissen oder erwähnte Texte zu kennen. Man bekommt ein Gespür für Argumentationsstrukturen, erkennt vielleicht Muster und Strategien in der Art und Weise, wie die unterschiedlichen Sprecher:innen Fragen stellen, monologisieren oder welche Begriffe sie am häufigsten verwenden.

Hendriks \& Kienhues (2019) werfen in einem Artikel zu Wissenschaftskom-

21 Vgl. etwa Fox \& Llinares (2016) um Podcasten als „disruptive academic practice“ und Klenks „Sprechendes Denken“ (2020). 
munikation den Begriff der ,scientific literacy' auf, der ebenfalls eng zusammenhängt mit der Möglichkeit, Menschen am Wissensbildungsprozess teilhaben zu lassen und sie dadurch zu informierten Einordnungen wissenschaftlicher Forschungen zu ermächtigen. Sie betonen dabei die gestiegene Bedeutung von Prozesswissen, also beispielsweise das Wissen über Forschungspraktiken (ebd., S. 36).

Genau dieses Prozesswissen, die Art, wie Argumente aufgebaut werden, Theorien, Zitate und historische Kontexte miteinander verknüpft werden können, wird in Podcasts auf besondere Weise vermittelt. Jemand, der nichts mit einer bestimmten fachlichen Disziplin, oder auch grundsätzlich nicht unmittelbar mit Forschung zu tun hat, wird in den seltensten Fällen einen wissenschaftlichen Aufsatz zur Hand nehmen, der darüber hinaus mehrfach überarbeitet und durchwirkt von akademischem Jargon ist. All die in solchen Artikeln getilgten Bestandteile des Prozesswissens, der Schleifen, Wortwiederholungen und Unsicherheiten sind Teil der mündlichen und freien Form in einem Podcast und können dazu beitragen, den Zugang zu akademischen Wissensprozessen zu erleichtern.

Darüber hinaus motivieren Podcasts aufgrund ihrer Niedrigschwelligkeit, des intimen Raums, in den man sich beim Zuhören versetzt fühlt und der Beziehung, die man zu Sprecher:innen aufbauen kann, eher zu Feedback, Rückmeldungen und Nachfragen. Dieses Potential beschreiben auch Birch und Weitkamp in Abgrenzung zum „one-way flow of information“: „Thus, new media, including podcasts, have the potential to bridge the divide between the unidirectional traditional media and face-to-face dialogue approaches, which may be limited by their audience reach." (Birch, 2010, S. 892) Insbesondere die Geisteswissenschaften können von diesem ,Brückenbau' profitieren und nahezu subversiv ihre Sichtbarkeit und mediale Repräsentation an traditionellen Medien vorbei erhöhen.

Neben dem aufgezeigten Potential von Podcasts, insbesondere auch die Zwischentöne, Ambivalenzen, Kontingenzen und individuellen Ausprägungen geisteswissenschaftlicher Forschungspraktiken und -haltungen hör- und nachvollziehbar zu machen, sind sie außerdem vielversprechend für den Abbau von Hürden für das Verständnis akademischer Praxis. Hier sei noch einmal betont, dass es dabei nicht in erster Linie um ein störungsfreies Nachvollziehen jahrhundertelang gewachsener Praktiken geht, sondern vielmehr um das Schaffen einer ,Plattform', eines Raums, der Praxis, Reflexion und, mit Klenk, „Darstellung als Durchführung“ verbindet.

Die hier ausgeführten Überlegungen sind als erste Ansätze zu verstehen und deswegen auch ein Versuch im Kleinen, zunächst an zwei literaturwissenschaftlichen Perspektiven und Podcastfolgen das Potential von Podcasts für die geisteswissenschaftliche Wissenschaftskommunikation auszuloten; sowie tentativ auch für die Beobachtung der Geisteswissenschaften und ihrer Praktiken, also für die Wissenschaftsforschung. Die eigenen Praktiken zu hinterfragen, zu reflektieren und zu analysieren, führt im Idealfall vielleicht auch zu neuen Impulsen für die Wissenschaftskommunikation. 
Dieser Ansatz kann als Grundlage für eine weitere Auseinandersetzung mit der auditiven Wissensvermittlung in den Geisteswissenschaften fruchtbar gemacht und auf andere Disziplinen übertragen werden. Der Fokus hier galt den häufig als sehr hermetisch wahrgenommenen Geisteswissenschaften, und insbesondere der Literaturwissenschaft, deren ,Black Box' der Praktiken die vorgestellten Podcastfolgen vielleicht etwas transparenter haben werden lassen.

\section{Literatur}

Albrecht, A., Danneberg, L., Krämer, O. \& Spoerhase, C. (2015). In Dies (Hrsg.), Theorien, Praktiken und Methoden des Interpretierens (S. 1-20,). Einleitung: de Gruyter. https://doi.org/10.1515/9783110353983.

Alt, P.-A. (2007).. Die Verheißungen der Philologie. Göttingen. Wallstein.

Behrs, J., Gittel, B. \& Klausnitzer, R. (2013). Wissenstransfer. Konditionen, Praktiken, Verlaufsformen der Weitergabe von Erkenntnis. Frankfurt a.M. https://doi.org/10.3726/978-3-653-03531-5.

Berlin, D. N. (2018). Episode 1, Wen kümmert's, wer spricht? (R. Kleist, Übers.). Verfügbar unter: https://dasneue.berlin/2018/03/22/dnb1-allmaehlichesverfertigen/

Birch, E., Hayley und Weitkamp. (2010). Podologues: conversations created by science podcasts. New Media and Society, 12(6), 889-909. https://doi.org/10.1177/1461444809356333.

Bromme, D., Rainer und Kienhues. (2014). In T. Seidel \& A. Krapp (Hrsg.), Pädagogische Psychologie (S. 55-81,). Weinheim. Beltz: Wissenschaftsverständnis und Wissenschaftskommunikation.

Fox, N. \& Llinares, D. (2016). Knowing Sounds: Podcasting as Academic Practice. Online-Podcast.

Geier, A. \& Gottschling, M. (2019). Wissenschaftskommunikation auf Twitter? Eine Chance für die Geisteswissenschaften! Mitteilungen des Deutschen Germanistenverbandes, 3(19), 282-292. https://doi.org/10.14220/mdge.2019.66.3.282.

Grasberger, L. (2016). Der Ton macht die Wissenschaft. Verfügbar unter: Online-Publikation:

Groth, S. K. \& Samson, K. (2016). Audio Papers - a Manifesto, Seismograf August 2016. Verfügbar unter: Online-Publikation:

Habermas, J. (1973). Erkenntnis und Interesse. Frankfurt a.M. Suhrkamp.

Hamann, J. (2014). Die Bildung der Geisteswissenschaften: Zur Genese einer sozialen Konstruktion zwischen Diskurs und Feld. Konstanz: UVK. 
Hanel, T. (1994). Naturwissenschaften und Technologie im Fernsehen des deutschsprachigen Raumes: TV-Wissenschaftsmagazine im Vergleich. Dissertation. München.

Hendriks, F. \& Kienhues, D. (2019). Science understanding between scientific literacy and trust: contributions from psychological and educational research. https://doi.org/10.1515/9783110255522-002.

Huber, L. (2019). Forschendes Lernen in den Geisteswissenschaften. Fernes Echo seiner historischen Ursprünge. In M.E. Kaufmann, A. Satilmis \& H.A. Mieg (Hrsg.), Forschendes Lernen in den Geisteswissenschaften. Konzepte, Praktiken und Perspektiven hermeneutischer Fächer (S. 21-33 ,). Wiesbaden. https://doi.org/10.1007/978-3-658-21738-9_2.

Kaufmann, M. E., Satilmis, A. \& Mieg, H. A. (2019). Einleitung: Forschendes Lernen in den Geisteswissenschaften. In Ansätze, Impulse und Herausforderungen, S. 1-18, in: Dies. (Hg.): Forschendes Lernen in den Geisteswissenschaften. Konzepte, Praktiken und Perspektiven hermeneutischer Fächer. Wiesbaden. https://doi.org/10.1007/978-3-658-21738-9_1.

Kleist, H. von. (о. J.). Über die allmähliche Verfertigung der Gedanken beim Reden. (von S. Ders., Hrsg.) (S. 319-324 ,). Helmut: Sämtliche Werke und Briefe.

Klenk, M. (2020). Sprechendes Denken. Essays zu einer experimentellen Kulturwissenschaft. Bielefeld. https://doi.org/10.14361/9783839452622.

Knorr-Cetina, K. (1981). The Manufacture of Knowledge: An Essay in the Constructivist and Contextual Nature of Science. Oxford. Pergamon.

Krause, M., Pethes, N. \& Krause. (2017). Scholars in Action. Deutsche Vierteljahrsschrift für Literaturwissenschaft und Geistesgeschichte, 91, 73-108. https://doi.org/10.1007/s41245-017-0032-4.

Latour, B. \& Woolgar, S. (1987). Laboratory Life: the Social Construction of Scientific Facts. Princeton. Princeton University Press. https://doi.org/10.1515/ 9781400820412 .

Martus, S. (2015). Wandernde Praktiken „,after theory"?, Internationales Archiv für Sozialgeschichte der deutschen Literatur. https://doi.org/10.1515/iasl2015-0010.

Martus, S. \& Spoerhase, C. (2009). Praxeologie der Literaturwissenschaft. Geschichte der Germanistik, 35(36), 89-96.

Milde, J. (2009). Vermitteln und Verstehen. In Zur Verständlichkeit von Wissenschaftsfilmen im Fernsehen. Wiesbaden. VS Verlag. DOI. https://doi.org/10.1007/ 978-3-531-91630-9.

Porombka, S. \& Schulze, H. (2018). In David \& N. Menzel (Hrsg.), „Eine Textur, in der das ,Textgerede' als Produktionssystem erscheint". Ein Gespräch über Audio Paper, S. 267-281, in: Assmann.https://doi.org/10.30965/9783770563876_017. 
Reckwitz, A. (2003). Grundelemente einer Theorie sozialer Praktiken. Eine sozialtheoretische Perspektive, Zeitschrift für Soziologie, 32(4), 282-301.

Schäfer, M. S. (2018). Geisteswissenschaften in den Medien. Ein Überblick über Studien zur medialen Repräsentation der Geisteswissenschaften. In M. Luginbühl \& J. Schröter (Hrsg.), Geisteswissenschaften und Öffentlichkeit - linguistisch betrachtet (S. 17-38 ,). Bern. https://doi.org/10.3726/b14151.

Scheu, A. M. \& Volpers, A.-M. (2017). Sozial- und Geisteswissenschaften im öffentlichen Diskurs. In H. Bonfadelli, B. Fähnrich, C. Lüthje, J. Milde, M. Rhomberg \& M.S. Schäfer (Hrsg.), Forschungsfeld Wissenschaftskommunikation (S. 391-404). Wiesbaden: Springer Fachmedien Wiesbaden. https://doi.org/10.1007/978-3-658-12898-2_21

Schruhl, F. (2018). Objektumgangsnormen in der Literaturwissenschaft. In M. Huber \& S. Krämer (Hrsg.), Wie Digitalität die Geisteswissenschaften verändert. Neue Forschungsgegenstände und Methoden. Online-Publikation. https://doi.org/10.17175/sbo03_012.

Snow, C. P. (1988). The two cultures and a second look; an expanded version of The two cultures and the scientific revolution. Cambridge: Cambridge University Press.

Spoerhase, C. (2015). In D. Andrea, K. Lutz, Olav \& C. Spoerhase (Hrsg.), Das „Laboratorium“ der Philologie? Das philologische Seminar als Raum der Vermittlung von Praxiswissen, S. 53-80, in: Albrecht. Berlin. https://doi.org/10.1515/ 9783110353983.53.

Theoretisch, P. (2019). Folge 12, Lernen wir aus Literatur und wozu eigentlich Literaturwissenschaft? Gespräch mit dem Literaturwissenschaftler Lutz Graner. 13.11.2019. Online-Podcast.

Weitze, M.-D. \& Heckl, W. M. (2016). Wissenschaftskommunikation - Schlüsselideen, Akteure, Fallbeispiele. Berlin/Heidelberg. https://doi.org/10.1007/ 978-3-662-47843-1.

Wissenschaft im Dialog/Kantar Emnid, 2019, Wissenschaftsbarometer 2019, Online-Publikation. (o. J.).. Verfügbar unter: https://www.wissenschaft-imdialog.de/projekte/wissenschaftsbarometer/wissenschaftsbarometer-2019 